\title{
Change in Red Cell Distribution Width as Predictor of Death and Neurologic Outcome in Patients Treated with Therapeutic Hypothermia after Out-of-Hospital Cardiac Arrest
}

Seongtak Kim, M.D., Jinseong Cho, M.D., Yongsu Lim, M.D., Jinjoo Kim, M.D., Hyukjun Yang, M.D., and Gun Lee, M.D.

Emergency Medicine, Gil Medical Center, Gachon University, Incheon, Korea

Background: The prognostic significance of change in red cell distribution width (RDW) during hospital stays in patients treated with therapeutic hypothermia (TH) after out-of-hospital cardiac arrest (OHCA) was investigated.

Methods: Patients treated with TH after OHCA between January 2009 and August 2013 were reviewed. Patients with return of spontaneous circulation (ROSC) were assessed according to Utstein Style. Hematologic variables including RDW, hematocrit, white blood cell count, and platelets were also obtained. RDW changes during the 72 hours after ROSC were categorized into five groups as follows: Group 1 (-0.8-0.1\%), Group 2 (0.2-0.3\%), Group 3 (0.4-0.5\%), Group 4 (0.6-0.8\%), and Group 5 ( $>0.8 \%)$.

Results: A total of 218 patients were enrolled in the study. RDW changes during the 72 hours after ROSC in Group 4 (HR $3.56,95 \%$ CI 1.25-10.20) and Group 5 (HR 5.07, 95\% CI 1.73-14.89) were associated with a statistically significant difference in one-month mortality. RDW changes were associated with statistically significant differences in neurologic outcome at 6 months after ROSC (Group 3 [HR 2.45, 95\% CI 1.17-5.14], Group 4 [HR 2.79, 95\% CI 1.33-5.84], Group 5 [HR 3.50, 95\% CI 1.35-7.41]). Other significant variables were location of arrest, cause of arrest, serum albumin, and advanced cardiac life support time.

Conclusions: RDW change during the 72 hours after ROSC is a predictor of mortality and neurologic outcome in patients treated with TH after OHCA.

Key Words: erythrocyte indices; mortality; out-of-hospital cardiac arrest; prognosis.

\section{Introduction}

The number of people who suffer cardiac arrest (CA) outside a hospital has increased recently. In 2012, there were 359,400 out-of-hospital cardiac arrests (OHCA) in the U.S, and only $9.5 \%$ of these patients survived.[1] It was also reported that only $7-10 \%$ of OHCA patients regained normal brain function[2,3] and that more than $90 \%$ ended up developing neuro-

Received on July 17, 2014

Revised on August 15, 2014

Accepted on August 20, 2014

Correspondence to: Jinseong Cho, Emergency Medicine, Gil Medical Center, Gachon University, 774-21 Namdongdae-ro, Namdong-gu, Incheon 405-760, Korea

Tel: +82-32-460-3015, Fax: +82-32-460-3019

E-mail: truecho@hanmail.net

ORCID: http://orcid.org/0000-0001-6762-4692

* No potential conflict of interest relevant to this article was reported. logical impairment or dying.[4] Even patients who achieve return of spontaneous circulation (ROSC) may experience post-cardiac arrest syndrome, which is a pathophysiological process that affects multiple organs.[5] While poor outcomes after CA pose a significant challenge, many studies have explored variables associated with mortality rate and neurological outcome after ROSC.[6-10]

The red cell distribution width (RDW) measures the variation of red blood cell size and is included in a complete blood count (CBC) laboratory test. RDW is useful in differentiating type of anemia[11,12] and is considered a strong prognostic marker for death caused by various diseases, including cardiovascular disease and inflammation. A high RDW value is also characteristic of CA, indicating a generally deteriorated systemic condition. Several previous studies have suggested that RDW is a prognostic marker for early death in trauma patients and OHCA patients; $[13,14]$ however, there is not enough evi- 
dence to support their claims, and studies investigating the association of RDW with neurological outcome are very rare. The aim of this study was to explore the association of changes in RDW with one-month mortality rate and neurological outcome at 6 months after ROSC in OHCA patients treated with therapeutic hypothermia (TH).

\section{Materials and Methods}

\section{1) Subjects}

This study was performed at a tertiary university hospital in Korea with 90,000 emergency department visits per year and nearly 100 intensive care unit beds. This study was conducted on patients who underwent TH after ROSC among those admitted to the emergency department due to CA from January 2009 to August 2013 (56 months). Of those treated with TH, survivors of CA for at least 24 hours were included, while pregnant women, children under 15 years, and patients whose families did not consent to treatment were excluded.

\section{2) Methods}

Patient information was collected using an in-hospital cardiac arrest registry based on Utstein style. All subjects had a complete blood count (CBC) drawn in the emergency department. RDW was routinely measured in subjects as part of the CBC at 24, 48, and 72 hours from ROSC, and the levels of electrolyte and pro-brain type natriuretic peptide (pro-BNP) and other components of blood were also measured. When subjects had indications for receiving TH after ROSC, informed consent was collected, and TH was performed according to the American heart association (AHA) guidelines and an in-hospital $\mathrm{TH}$ protocol. Subjects with ROSC were rapidly cooled to $33^{\circ} \mathrm{C}$ or $34^{\circ} \mathrm{C}$ using surface or endovascular techniques, and then their body temperature was maintained at these target temperatures for 24 hours before rewarming began at a rate of 0.2 to $0.3^{\circ} \mathrm{C}$ per hour.

\section{3) Subject variables}

We analyzed demographic characteristics of subjects, location of CA, witness status, initial cardiac rhythm, subsequent cardiac rhythm, causes of CA, acute physiology and chronic health evaluation II (APACHE II) score, and RDW at baseline, 24, 48, and 72 hours after ROSC. Changes in RDW values for 72 hours were defined as explanatory variables.

\section{4) Mortality and neurological outcome}

We examined mortality within one month and neurological outcome at 6 months after ROSC. As shown in previous studies, the primary endpoint was early mortality, which was defined as death within one month in this study. Neurological outcome at 6 months was used as the secondary endpoint due to its reported usefulness in predicting long-term clinical outcomes. Cerebral performance category (CPC) score was used to assess neurological outcome on a 5-point scale: CPC 1 means good cerebral performance; 2 means moderate cerebral disability with independence in daily life; 3 means severe cerebral disability, conscious but dependent due to unstable cerebral function; 4 means coma or persistent vegetative state; and 5 means death. CPC 1-2 is classified as "good neurological outcome," and CPC 3-5 is classified as "poor neurological outcome."

\section{5) Statistical analysis}

All statistical analysis was performed using SPSS Statistics 18 (IBM corp., Inc., Chicago, IL, USA). Chi-square test was used for categorical variables, and Mann-Whitney $U$ test was used for continuous variables that were not normally distributed. Cox proportional hazards regression model was used for multivariate analysis of mortality and neurological outcome, and the model was retrospectively adjusted. All tests for statistical significance were conducted by computing a $95 \%$ two-sided confidence interval.

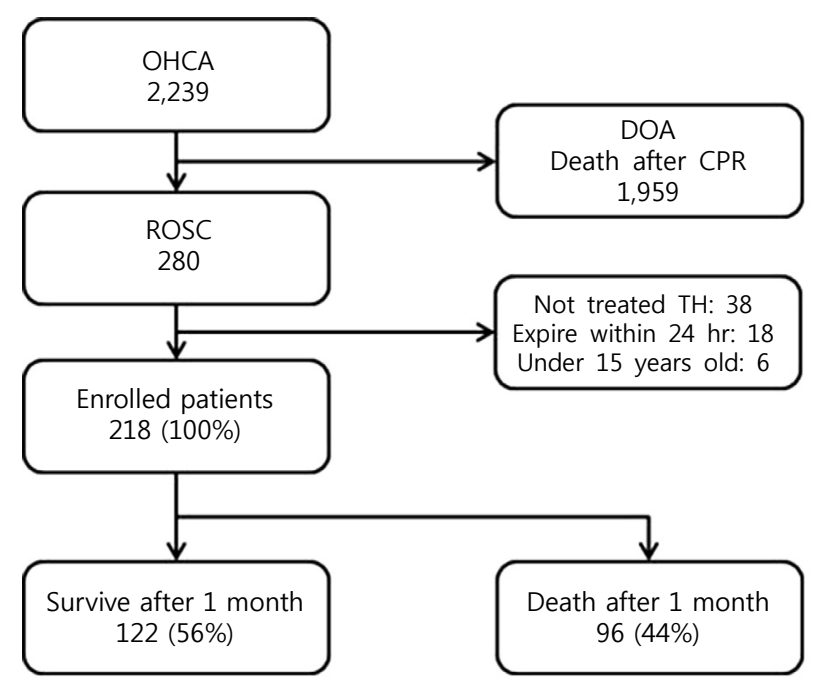

Fig. 1. Study subject flow diagram. There were 2,239 patients admitted for OHCA from January 2009 to August 2013. 280 patients achieved ROSC. 62 patients were excluded because they were not treated with $\mathrm{TH}$, they died within 24 hours, or they were younger than 15 years. A total of 218 patients were included in the final analysis. $122(56 \%)$ patients survived at one month, while $96(44 \%)$ patients died. OHCA: out-of-hospital cardiac arrest; DOA: death on arrival; CPR: cardiopulmonary resuscitation; ROSC: return of spontaneous circulation; $\mathrm{TH}$ : therapeutic hypothermia. 

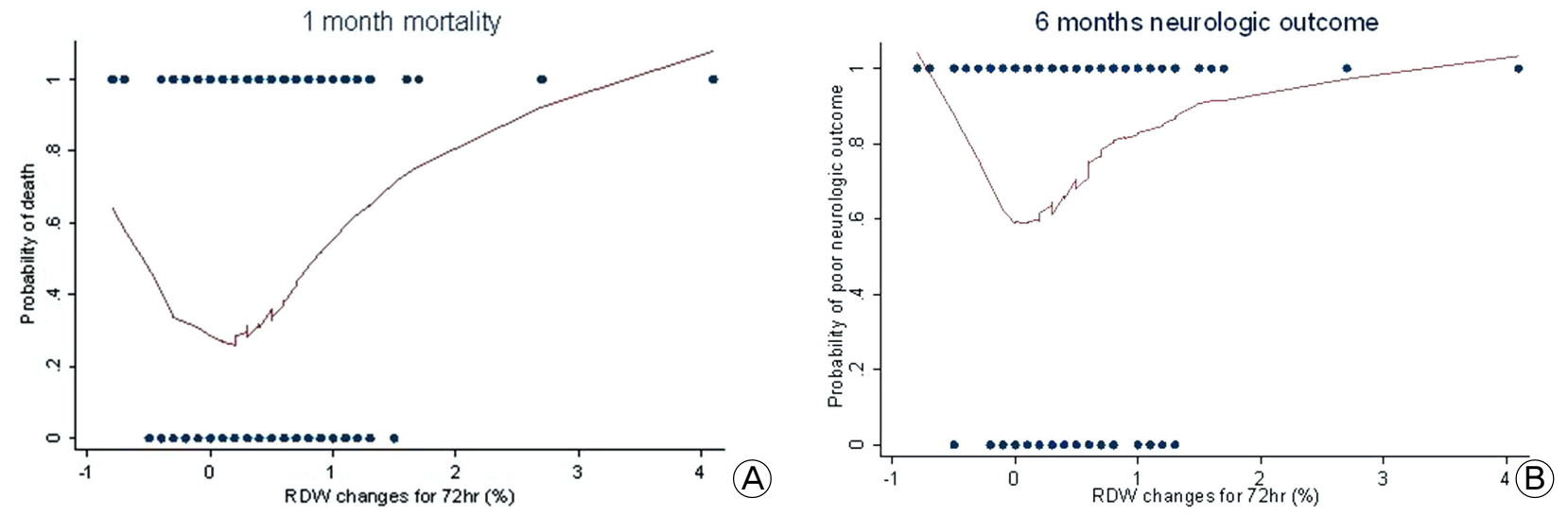

Fig. 2. Probability of one-month mortality and 6 months neurologic outcome according to RDW changes for 72 hours after ROSC. (A) This graph shows one-month mortality according to RDW changes for 72 hours after ROSC. Increased or decreased RDW changes in both directions seem to increase mortality. (B) This graph shows the 6-month neurologic outcome according to RDW changes for 72 hours after ROSC. Increased or decreased RDW changes in both directions seem to increase incidence of poor neurologic outcome. RDW: red cell distribution width; ROSC: return of spontaneous circulation.

Table 1. Baseline characteristics of study population $(\mathrm{n}=218)$

\begin{tabular}{lc}
\hline \hline Variables & Median (IQR) \\
\hline Male sex, $\mathrm{n}(\%)$ & $149(68.3)$ \\
Age, yr (mean \pm SD) & $51.6 \pm 14.9$ \\
Location, $\mathrm{n}(\%)$ & \\
Home & $116(53.2)$ \\
Public & $96(44.0)$ \\
Other & $6(2.8)$ \\
Bystander & $72(33.0)$ \\
Shockable rhythm, n (\%) & $67(30.7)$ \\
Cardiogenic, $\mathrm{n}(\%)$ & $119(54.6)$ \\
Hb, g/dL & $14(12.5-15.4)$ \\
Hct, $\%$ & $41(36.7-44.6)$ \\
WBC, $\times 10^{3} / \mathrm{mm}^{3}$ & $12.36(9.8-16.0)$ \\
RDW, \% & $13(12.5-13.5)$ \\
RDW change during $72 \mathrm{hr}, \%$ & $0.4(0.2-0.7)$ \\
MCV, fl & $91.8(88.5-95)$ \\
MCH, pg & $31.4(30.2-32.5)$ \\
Plt, $\times 10^{3} / \mathrm{mm}$ & $217(177.5-253.5)$ \\
BUN, mg/dL & $15.8(12.3-20.8)$ \\
Cr, mg/dL & $1.1(0.9-1.3)$ \\
Alb, g/dL & $3.95(3.6-4.2)$ \\
S100 & $1.29(0.4-3.2)$ \\
lactate & $7.95(5.8-11.2)$ \\
Pro-BNP, pg/ml & $96.42(41.47-528.65)$ \\
APACHE II & $27(23-31)$ \\
BLS time, min & $6(2-10)$ \\
ACLS time, min & $20(15-27)$ \\
1-month mortality, n (\%) & $96(44.0)$ \\
6-month CPC, n (\%) & \\
Good & $60(27.5)$ \\
Poor & $158(72.5)$ \\
\hline & \\
\hline &
\end{tabular}

IQR: interquartile range; $\mathrm{Hb}$ : hemoglobin; Hct: hematocrit; WBC: white blood cell; RDW: red cell distribution width; MCV: mean corpuscular volume; fl: femtoliters; pg: picogram; Plt: platelet; BUN: blood urea nitrogen; Cr: creatinine; Alb: albumin; BNP: B-type natriuretic peptide; APACHE: acute physiology and chronic health evaluation; BLS: basic life support; ACLS: advanced cardiac life support; CPC: cerebral performance category.

\section{Results}

\section{1) Characteristics of subjects}

A total of 2,239 patients presented with OHCA from January 2009 to August 2013. Of those, 260 achieved ROSC. Patients were excluded if they did not undergo TH, if they died within 24 hours, or if they were under the age of 15 years. The remaining 218 subjects were analyzed (Fig. 1).

There were 149 men (68.3\%) and 69 women (31.7\%) with a median age of 51.5 years. Only 72 of $218(33.0 \%)$ subjects received bystander cardiopulmonary resuscitation (CPR). Baseline RDW values were median $13.0 \%$ with interquartile range (IQR) $12.5-13.5 \%$. Changes in RDW during the first 72 hours after ROC were median $0.4 \%$ with IQR $0.2-0.7 \%$. Of the 218 subjects, $122(56 \%)$ survived one month, 96 (44\%) died, 60 (27.5\%) had a CPC score of 1 or 2 at 6 months, and 155 (72\%) had a CPC score of 3 or 4 (Table 1).

Variables significantly associated with one-month mortality included baseline electrocardiography (ECG) results, causes of CA, CA-basic life support (BLS) time, and CA-advanced cardiac life support (ACLS) time. In addition, baseline RDW value, change in RDW during the first 72 hours, albumin, S100, and APACHE II score were significantly associated with early mortality. Variables that were significantly associated with neurological outcome at 6 months included baseline ECG results, causes of CA, CA-BLS time, CA-ACLS time, age, and sex. According to $\mathrm{CBC}$ results, baseline RDW, change in RDW during the first 72 hours, hemoglobin, albumin, S100, and APACHE-II score were also significantly associated with neurological outcome (Table 2). 
316 The Korean Journal of Critical Care Medicine: Vol. 29, No. 4, November 2014

Table 2. Variables by mortality at 1 month and neurologic outcome at 6 months after ROSC $(\mathrm{N}=218)$

\begin{tabular}{|c|c|c|c|c|c|c|}
\hline \multirow[b]{2}{*}{ Variables } & \multicolumn{3}{|c|}{ 1-month mortality, n (\%) } & \multicolumn{3}{|c|}{ 6-month neurologic outcome, $\mathrm{n}(\%)$} \\
\hline & $\begin{array}{l}\text { Survived } \\
(\mathrm{n}=122)\end{array}$ & $\begin{array}{c}\text { Death } \\
(\mathrm{n}=96)\end{array}$ & $\mathrm{p}$ value & $\begin{array}{c}\text { Good } \\
(\mathrm{n}=60)\end{array}$ & $\begin{array}{c}\text { Poor } \\
(\mathrm{n}=158)\end{array}$ & $\mathrm{p}$ value \\
\hline Male sex & $89(73.0)$ & $60(62.5)$ & 0.100 & $49(81.7)$ & $100(63.3)$ & 0.009 \\
\hline Age, yr (mean \pm SD) & $50.3 \pm 14.1$ & $53.3 \pm 15.7$ & 0.141 & $47.1 \pm 11.7$ & $53.3 \pm 15.6$ & 0.007 \\
\hline Location & & & 0.073 & & & 0.154 \\
\hline Home & $60(50.8)$ & $54(56.3)$ & & $29(48.3)$ & $87(55.1)$ & \\
\hline Public & $59(48.4)$ & $37(38.5)$ & & $31(51.7)$ & $65(41.1)$ & \\
\hline Other & $1(0.8)$ & $5(5.2)$ & & 0 & $6(3.8)$ & \\
\hline Bystander & $46(37.7)$ & $26(27.1)$ & 0.098 & $23(38.3)$ & $49(31)$ & 0.305 \\
\hline Shockable rhythm & $51(41.8)$ & $16(16.7)$ & $<0.001$ & $36(60)$ & $31(19.6)$ & $<0.001$ \\
\hline Cardiogenic & $84(68.9)$ & $35(36.5)$ & $<0.001$ & $56(93.3)$ & $63(39.9)$ & $<0.001$ \\
\hline BLS time, median (IQR) & $6(1-9)$ & $7(4-12)$ & 0.080 & $5(0-9)$ & $7(4-12)$ & 0.007 \\
\hline ACLS time, median (IQR) & $19(14-24)$ & $23(18-30)$ & $<0.001$ & $19(13-22)$ & $21(16-29)$ & 0.013 \\
\hline RDW, \% & $12.9(12.4-13.4)$ & $13.1(12.6-13.7)$ & 0.009 & $12.7(12.3-13.2)$ & $13.1(12.6-13.6)$ & $<0.001$ \\
\hline RDW change during $72 \mathrm{hr}, \%$ & $0.3(0.1-0.5)$ & $0.6(0.2-0.9)$ & 0.002 & $0.3(0.1-0.5)$ & $0.4(0.2-0.8)$ & 0.003 \\
\hline $\mathrm{Hb}, \mathrm{g} / \mathrm{dL}$ & $14.3(13.3-15.8)$ & $13.3(11.3-15.0)$ & $<0.001$ & $14.9(13.7-16.4)$ & $13.5(11.7-15.0)$ & $<0.001$ \\
\hline $\mathrm{Alb}, \mathrm{g} / \mathrm{dL}$ & $4.0(3.8-4.2)$ & $3.8(3.4-4.1)$ & $<0.001$ & $4.1(3.8-4.3)$ & $3.9(3.5-4.2)$ & 0.002 \\
\hline $\mathrm{S} 100$ & $1.1(0.4-2.3)$ & $1.7(0.6-5.4)$ & 0.018 & $0.8(0.3-1.8)$ & $1.5(0.6-4.0)$ & 0.001 \\
\hline APACHE II & $26(23-29)$ & $29(26-33)$ & $<0.001$ & $26(22-29)$ & $28(24-32)$ & 0.003 \\
\hline
\end{tabular}

ROSC: return of spontaneous circulation; SD: standard deviation; BLS: basic life support; IQR: interquartile range; ACLS: advanced cardiac life support; RDW: red cell distribution width; Hb: hemoglobin; Alb: albumin; APACHE: acute physiology and chronic health evaluation.

\section{2) Analysis of change in red cell distribution width}

The relationship between RDW change and mortality and neurological outcome is U-shaped (Fig. 2). The bidirectional effects of RDW were divided into quintiles: quintile 1 represents $-0.8-0.1 \%$ RDW change, quintile 2 represents $0.2-0.3 \%$ RDW increase, quintile 3 represents $0.4-0.5 \%$ RDW increase, quintile 4 represents $0.6-0.8 \%$ RDW increase, and quintile 5 represents more than $0.8 \%$ RDW increase. Because mortality rate was lower in quintile 2 , statistical analysis was performed on the basis of quintile 2 .

\section{3) Multivariate analysis using Cox's regression model}

Multivariable Cox proportional hazards analysis was performed to assess the association between RDW change in 72 hours and one-month mortality rate. As a result, RDW change was statistically significant in quintile 4 (Hazard ratio [HR] $5.46,95 \%$ confidence interval [CI] 1.54-19.40) and quintile 5 (HR 6.99, 95\% CI 1.92-25.47). CA of non-cardiac origin, albumin, and CA-ACLS time were also statistically significant (Table 3).

The association of RDW change with neurological outcome at 6 months was statistically significant in quintile 3 (HR 2.45, 95\% CI 1.17-5.14), quintile 4 (HR 2.79, 95\% CI 1.33-5.84), and quintile 5 (HR $3.50,95 \%$ CI 1.35-7.41). CA of non-cardiac origin, albumin, and CA-ACLS time also affected neurological outcome (Table 4).

These top three quintiles also showed a significant relationship with neurological outcome at one month: quintile 3 (HR
Table 3. Multivariate cox proportional regression model of the association between predictor variables and mortality in PCAS patients at 1 month after ROSC

\begin{tabular}{|c|c|c|}
\hline & $\begin{array}{c}\text { Unadjusted hazard ratio } \\
(95 \% \mathrm{CI})\end{array}$ & $\begin{array}{c}\text { Adjusted hazard ratio } \\
(95 \% \mathrm{CI})\end{array}$ \\
\hline \multicolumn{3}{|l|}{ RDW changes } \\
\hline Group $1(-0.8-0.1)$ & $1.82(0.77-4.29)$ & $3.62(0.99-13.15)$ \\
\hline Group $2(0.2-0.3)$ & Ref. & Ref. \\
\hline Group $3(0.4-0.5)$ & $1.73(0.71-4.22)$ & $3.48(0.90-13.55)$ \\
\hline Group 4 (0.6-0.8) & $3.33(1.45-7.67)$ & $5.46(1.54-19.40)$ \\
\hline Group $5(>0.8)$ & $4.45(1.95-10.19)$ & $6.99(1.92-25.47)$ \\
\hline Age & $1.01(0.99-1.02)$ & - \\
\hline Female & $1.49(0.97-2.30)$ & - \\
\hline \multicolumn{3}{|l|}{ Rhythm } \\
\hline Shockable & Ref. & - \\
\hline Non-shockable & $2.71(1.53-4.81)$ & - \\
\hline \multicolumn{3}{|l|}{ Location } \\
\hline Home & Ref. & Ref. \\
\hline Public & $0.78(0.50-1.22)$ & $0.79(0.42-1.47)$ \\
\hline Other & $2.59(1.03-6.52)$ & $6.03(1.90-19.14)$ \\
\hline \multicolumn{3}{|l|}{ Bystander } \\
\hline Bystander & Ref. & - \\
\hline No-bystander & $1.31(0.82-2.08)$ & - \\
\hline \multicolumn{3}{|l|}{ Cause } \\
\hline Cardiac & Ref. & Ref. \\
\hline Non-cardiac & $2.80(1.79-4.37)$ & $3.01(1.58-5.73)$ \\
\hline $\mathrm{Hb}$ & $0.86(0.79-0.93)$ & - \\
\hline Alb & $0.46(0.31-0.68)$ & $0.52(0.29-0.94)$ \\
\hline S100 & $1.05(1.04-1.09)$ & $1.02(0.99-1.05)$ \\
\hline BLS time & $1.02(1.00-1.04)$ & $0.97(0.93-1.01)$ \\
\hline ACLS time & $1.03(1.01-1.04)$ & $1.04(1.01-1.06)$ \\
\hline APACHE II & $1.06(1.03-1.09)$ & $1.02(0.78-1.07)$ \\
\hline
\end{tabular}

PCAS: post-cardiac arrest syndrome; ROSC: return of spontaneous circulation; RDW: red cell distribution width; Hb: hemoglobin; Alb: albumin; BLS: basic life support; ACLS: advanced cardiac life support; APACHE: acute physiology and chronic health evaluation. 
Table 4. Multivariate cox proportional regression model of the association between predictor variables and neurologic outcome in PCAS patients at 6 months after ROSC

\begin{tabular}{|c|c|c|}
\hline & $\begin{array}{c}\text { Unadjusted hazard ratio } \\
(95 \% \mathrm{CI})\end{array}$ & $\begin{array}{c}\text { Adjusted hazard ratio } \\
(95 \% \mathrm{CI})\end{array}$ \\
\hline \multicolumn{3}{|l|}{ RDW changes } \\
\hline Group $1(-0.8-0.1)$ & $1.38(0.79-2.44)$ & $2.01(0.98-4.15)$ \\
\hline Group $2(0.2-0.3)$ & Ref. & Ref. \\
\hline Group $3(0.4-0.5)$ & $1.75(0.99-3.05)$ & $2.45(1.17-5.14)$ \\
\hline Group 4 (0.6-0.8) & $2.20(1.24-3.90)$ & $2.79(1.33-5.84)$ \\
\hline Group $5(>0.8)$ & $2.86(1.61-5.06)$ & $3.50(1.35-7.41)$ \\
\hline Age & $1.01(1.00-1.02)$ & $1.01(0.99-1.02)$ \\
\hline Female & $1.54(1.11-2.15)$ & - \\
\hline \multicolumn{3}{|l|}{ Rhythm } \\
\hline Shockable & Ref. & - \\
\hline Non-shockable & $2.54(1.69-3.82)$ & - \\
\hline \multicolumn{3}{|l|}{ Location } \\
\hline Home & Ref. & Ref. \\
\hline Public & $0.81(0.58-1.13)$ & $0.81(0.52-1.28)$ \\
\hline Other & $2.37(1.03-5.45)$ & $5.75(2.15-15.42)$ \\
\hline \multicolumn{3}{|l|}{ Bystander } \\
\hline Bystander & Ref. & - \\
\hline No-bystander & $1.20(0.85-1.69)$ & - \\
\hline \multicolumn{3}{|l|}{ Cause } \\
\hline Cardiac & Ref. & Ref. \\
\hline Non-cardiac & $2.86(2.05-4.00)$ & $2.85(1.85-4.41)$ \\
\hline $\mathrm{Hb}$ & $0.87(0.82-0.93)$ & - \\
\hline Alb & $0.54(040-0.74)$ & $0.64(0.41-0.99)$ \\
\hline S100 & $1.04(1.00-1.07)$ & $1.02(0.99-1.05)$ \\
\hline BLS time & $1.02(1.00-1.03)$ & $0.97(0.94-1.01)$ \\
\hline ACLS time & $1.02(1.00-1.03)$ & $1.03(1.01-1.06)$ \\
\hline APACHE II & $1.05(1.03-1.07)$ & $1.02(0.99-1.05)$ \\
\hline
\end{tabular}

PCAS: post-cardiac arrest syndrome; ROSC: return of spontaneous circulation; RDW: red cell distribution width; Hb: hemoglobin; Alb: albumin; BLS: basic life support; ACLS: advanced cardiac life support; APACHE: acute physiology and chronic health evaluation.

2.47 and $95 \%$ CI 1.19-5.15), quintile 4 (HR 2.93 and 95\% CI 1.41-6.11), and quintile 5 (HR 3.53 and 95\% CI 1.66-7.52).

Quintile 1, which contained negative changes in RDW, had no statistically significant effect on either mortality or neurological outcome.

\section{Discussion}

This study ascertains the efficacy of RDW change during the first 72 hours after ROSC as a predictor of mortality within one month and neurological outcome at 6 months. Currently, there is no mechanism that clearly explains the association between RDW change and mortality and neurological outcome. Some previous studies have described baseline RDW values as prognostic markers of early mortality in patients with critical illness, trauma, and OHCA. In other words, RDW level is likely to reflect the overall condition after $\mathrm{CA}$ and therefore is predictive of outcomes. In a previous study assessing the relationship between baseline RDW and mortality within one month in OHCA patients, the highest quartile showed a significant difference with HR 1.95 (95\% CI 1.05-3.60, p = 0.034), compared with the lowest quartile.[14] Although the connection with neurological outcome was not discussed in that study, this study identified the association of RDW with neurological outcome by analyzing the impacts of baseline RDW and changes in RDW during 72 hours. No significant impact was found in the former, whereas a statistically significant impact was found in the latter.

Several studies have addressed the role of RDW changes in predicting outcomes. One of them reported that RDW was significantly increased by 0.58 at 72 hours after bacteremia, compared with baseline values $(\mathrm{p}=0.01)$.[15] Another study reported that RDW changes during 72 hours were significantly meaningful factors in predicting mortality at 28 days in septic patients.[16] Thus, the effect of RDW changes during 72 hours appears to be consistent across studies. This study, however, found no significant impact of RDW changes during the first 24 and 48 hours.

Several studies addressed the effectiveness of RDW as a prognostic marker in patients with critical illness. RDW was significantly effective in the prediction of mortality at 30 days in patients with community-acquired pneumonia.[17] RDW was reported as a strong independent prognostic marker of death from all causes in patients who underwent coronary angiogram (HR 2.69, 95\% CI 1.50-4.84, p < 0.01).[18] RDW was the second most effective variable in predicting survival rate within one year (next to NT-pro-BNP) in patients with renal failure.[19] Finally, the highest quartile of RDW was significantly associated with mortality from all causes in one population.[20]

Most studies were focused on the association between baseline RDW and mortality. This study, however, included neurological outcome at 6 months and looked at changes in RDW over time, making its results worthwhile.

This study has the following limitations. First, patients at a university hospital are not a random sample from the population of OHCA patients, and the results cannot be generalized to the larger population. Second, the sample size is not large enough to ensure statistical significance. Third, therapeutic hypothermia was one of the most important inclusion criteria in this study; therefore, it does not include any clinical data on patients who did not undergo TH. Even so, TH is widely used around the world to assure a more favorable outcome for patients with post-cardiac arrest syndrome. Any valid way to pre- 
dict outcomes in patients after TH is useful. Finally, because RDW changes were assessed for up to 72 hours after ROSC, there is no data and discussion on further changes in RDW after 72 hours.

Changes in RDW during the first 72 hours after ROSC were useful for predicting mortality within one month and neurological outcome at 6 months in OHCA patients. It is clinically worthwhile to demonstrate the prognostic ability of the cost-effective $\mathrm{CBC}$ in predicting early mortality and long-term neurological outcomes. Further prospective multicenter studies are needed to confirm this finding.

\section{References}

1) Go AS, Mozaffarian D, Roger VL, Benjamin EJ, Berry JD, Borden WB, et al: Heart disease and stroke statistics--2013 update: a report from the American heart association. Circulation 2013; 127: e1-240.

2) Pell JP, Sirel JM, Marsden AK, Ford I, Walker NL, Cobbe SM: Presentation, management, and outcome of out of hospital cardiopulmonary arrest: comparison by underlying aetiology. Heart 2003; 89: 839-42.

3) Atwood C, Eisenberg MS, Herlitz J, Rea TD: Incidence of EMS-treated out-of-hospital cardiac arrest in Europe. Resuscitation 2005; 67: 75-80.

4) Temple A, Porter R: Predicting neurological outcome and survival after cardiac arrest. Continuing Education in Anaesthesia, Critical Care \& Pain [serial on the internet]. 2012 May [2012 May 21]. Available from http://ceaccp.oxford journals.org/content/early/2012/05/20/bjaceaccp.mks029.full

5) Neumar RW, Nolan JP, Adrie C, Aibiki M, Berg RA, Böttige $\mathrm{BW}$, et al: Post-cardiac arrest syndrome: epidemiology, pathophysiology, treatment, and prognostication. A consensus statement from the International liaison committee on resuscitation (American heart association, Australian and New Zealand Council on resuscitation, European resuscitation council, heart and stroke foundation of Canada, InterAmerican heart foundation, resuscitation council of Asia, and the resuscitation council of Southern Africa); the American heart association emergency cardiovascular care committee; the council on cardiovascular surgery and anesthesia; the council on cardiopulmonary, perioperative, and critical care; the council on clinical cardiology; and the stroke council. Circulation 2008; 118: 2452-83.

6) Peberdy MA, Callaway CW, Neumar RW, Geocadin RG, Zimmerman JL, Donnino M, et al: Part 9: post-cardiac arrest care 2010 American heart association guidelines for cardiopulmonary resuscitation and emergency cardiovascular care. Circulation 2010; 122: S768-86.

7) Sasson C, Rogers MA, Dahl J, Kellermann AL: Predictors of survival from out-of-hospital cardiac arrest: a systematic review and meta-analysis. Circ Cardiovasc Qual Outcomes 2010; 3: 63-81.

8) Fugate JE, Wijdicks EF, Mandrekar J, Claassen DO, Manno EM, White RD, et al: Predictors of neurologic outcome in hypothermia after cardiac arrest. Ann Neurol 2010; 68: 907-14.

9) Sandroni C, Nolan J, Cavallaro F, Antonelli M: In-hospital cardiac arrest: incidence, prognosis and possible measures to improve survival. Intensive Care Med 2007; 33: 237-45.

10) Scolletta S, Donadello K, Santonocito C, Franchi F, Taccone FS: Biomarkers as predictors of outcome after cardiac arrest. Expert Rev Clin Pharmacol 2012; 5: 687-99.

11) Morris M, Davey FR: Basic examination of blood. In: Clinical diagnosis and management by laboratory methods. 20th ed. Edited by Henry JB: Philadelphia, W.B. Saunders Company. 2001, 544-5.

12) Demir A, Yarali N, Fisgin T, Duru F, Kara A: Most reliable indices in differentiation between thalassemia and trait and iron deficiency anemia. Pediatr Int 2002; 44: 612-6.

13) Majercik S, Fox J, Knight S, Horne BD: Red cell distribution width is predictive of mortality in trauma patients. J Trauma Acute Care Surg 2013; 74: 1021-6.

14) Kim J, Kim K, Lee JH, Jo YH, Rhee JE, Kim TY, et al: Red blood cell distribution width as an independent predictor of all-cause mortality in out of hospital cardiac arrest. Resuscitation 2012; 83: 1248-52.

15) Lee JH, Chung HJ, Kim K, Jo YH, Rhee JE, Kim YJ, et al: Red cell distribution width as a prognostic marker in patients with community-acquired pneumonia. Am J Emerg Med 2013; 31: 72-9.

16) Slaughter E, Mount C, Schofield C: Changes in red blood cell width distribution as a novel biomarker for bacteremia. Crit Care Med 2013; 41: A259.

17) Kim CH, Park JT, Kim EJ, Han JH, Han JS, Choi JY, et al: An increase in red blood cell distribution width from baseline predicts mortality in patients with severe sepsis or septic shock. Critical Care [serial on the internet]. 2013 Dec [2013 Dec 9]. Available from http://ccforum.com/content/17/6/R282

18) Cavusoglu E, Chopra V, Gupta A, Battala VR, Poludasu S, Eng C, et al: Relation between red blood cell distribution width (RDW) and all-cause mortality at two years in an unselected population referred for coronary angiography. Int $\mathrm{J}$ 
Cardiol 2010; 141: 141-6.

19) Al-Najjar Y, Goode KM, Zhang J, Cleland JG, Clark AL: Red cell distribution width: an inexpensive and powerful prognostic marker in heart failure. Eur J Heart Fail 2009; 11: 1155-62.
20) Chen PC, Sung FC, Chien KL, Hsu HC, Su TC, Lee YT: Red blood cell distribution width and risk of cardiovascular events and mortality in a community cohort in Taiwan. Am J Epidemiol 2010; 171: 214-20. 